\title{
Recovery Does Not Prevent Myocardial Damage Due to Overtraining (Biomolecular and Pathobiology Studies)
}

\author{
Made Kurnia Widiastuti Giri \\ Faculty of Sport Sciences \\ Universitas Pendidikan Ganesha \\ Singaraja, Bali, Indonesia \\ kurnia.widiastuti@undiksha.ac.id \\ Muchsin Doewes \\ Faculty of Sport Sciences \\ Universitas Sebelas Maret \\ Surakarta, Indonesia \\ mdoewes2000@yahoo.com
}

\author{
Ketut Indra Purnomo \\ Faculty of Sport Sciences \\ Universitas Pendidikan Ganesha \\ Singaraja, Bali, Indonesia \\ asalkode@yahoo.com \\ Herka Maya Jatmika \\ Faculty of Sport Sciences \\ Universitas Negeri Yogyakarta \\ Yogyakarta, Indonesia \\ herka_mayajatmika@uny.ac.id
}

\begin{abstract}
Overtraining the body with exercises and without adequate recovery time would lead to various health conditions. The overtraining conditions, usually leads to oxidative stress which damages the body tissue. This study aims to analyze the effect associated with a recovery duration of three and seven days in order to prevent myocardial damage due to overtraining. The research made use of a post experimental test of 32 rats which were divided into 4 groups namely 1) proportional sport, 2) overtraining, 3) overtraining with recovery of 3 days and 4) overtraining with recovery of 7 days. Histopathology examination was performed at the end of the experiment period on the rats. The results obtained has it that there were myocardial damage in the left ventricular hypertrophy, necrosis and chromatin condensation of these rats. Posttest comparative analysis was done to analyze and obtain a posttest mean for each group. For MDA myocardium concentration, Analysis Of Variance (ANOVA) results showed $F$ to be equal to 6,248 with $p=0,002$; SOD myocardium concentration was $F=19.519$ with $p=0.0001$; Left Ventricular Hypertrophy Index was $F=140,732$ with $p=.0,0001$; Index of myocardium necrosis between groups, was $F=201,911$ with $p=$ 0.0001; Chromatin condensation index on the cardiomyocyte between the study groups was $F=524,466$ with $p=0.0001$. Post hoc tests showed different significant concentration at MDA and SOD without any different in the left ventricular hypertrophy, necrosis and chromatin condensation. The results of this study proves that there is no effect inmyocardial damage as a result of overtraining from three and seven days, notwithstanding the fact that recovery decreases MDA myocardium concentrations, increases SOD myocardium concentrations, but does not prevent left ventricular hypertrophy, necrosis and chromatin condensation.
\end{abstract}

Keywords - recovery, myocardium damage, overtraining

\section{INTRODUCTION}

Overtraining or overloading exercises is often experienced when an athlete is preparing for a competition either with a limited timeframe or with adequate timeframe. Overtraining the body without adequate recovery periods, would lead to some myocardium damages on the athlete. According to [21] and [18], an athlete's body condition is very vulnerable if faced with overload dose of exercise without adequate rest at intervals.

Furthermore, during the period of overtraining training, Reactive Oxygen Species (ROS) is released from the body. This ROS released, exceeds the protective capacity of the endogenous antioxidant system and causes deregulations in the inflammatory system, oxidative phosphorylation and neuroendocrine [5]. Several studies has it that increased exercise intensity increases the oxidative stress and free radical production in cells [2].

Overtraining is a condition associated with oxidative stress which affects various organs of the body [18]. The study of the effects of overtraining on cardiac organ is debatable. However, in some studies on cardiac myositis, overtraining leads to pathological changes in the heart. Animal test studies was carried out to examine the causes of sudden death in athletes as a result of cardiac organ caused by the hypertrophy of cardiomyopathy. Hypertrophy of cardiomyopathy is defined as a congenital aberration, obtained through autopsy results which in some cases is one of the major causes of sudden cardiac death in athletes. This is said to be one of the causes of sudden cardiac death on athletes though this analysis is yet to be properly documented. Athletes are described as those individuals in a community that are more fit and healthy than non-athletes. The presence of sudden cardiac death in athletes has seized public attention. This is simply because it is a paradoxical ailment, leaving the public to assume the health status of the athletes [14]. Through autopsies performed in some cases of sudden cardiac death in athletes, cardiomyopathy hypertrophy is reported to be the major causes of sudden death in young athletes [8], [5], [10] However, in cases where autopsy was not conducted on the body of these young athletes, the cause of death cannot be ascertained. Similarly, experiment carried out using animals, has shown incorrect training to cause changes in heart rate and stroke volume of the heart. In this study, myocardium damage to histologic 
overtraining will be demonstrated on the basis of patho mechanism that begins with the formation of excessive ROS. Damage to myocardium which occurs as a result of overtraining is suspected to result in an increased risk of cardiovascular diseases. Proper training management to avoid overtraining is carried out by decreasing the training load and recovery time [12]. Based on previous researches, it is evident that ROS is an underlying etiology used to determine the body system adaptation to sport. However, its major failure in adaptation is patomechanism in cardiac organ, as well as the inability to effectively explain the effectiveness of the recovery period using some previous studies. Recovery will induce the body's hormonal ability to decrease the ROS in the system and enable it meet the production capacity of endogenous antioxidants, one of which is Superoxide Dismutase (SOD). SOD is an endogenous antioxidant formed by mitochondria. [1], [7], Detraining or recovery is defined as a management timeframe given in overtraining conditions. Recovery will restore the exhausted cells so that it can return to its homeostatic conditions [12], [4] . The basic recovery principle is the recovery process which is dependent on the type and duration of physical stress within the given training period [3]. The right recovery duration required by the body cells is explained through several recovery mechanisms studies that occur in the skeletal muscle. Recovery is intended to restore phosphor creatin, glycogen and to increase blood flow for oxygen consumption (reperfusion) [15], [9], [11]. The time required for the recovery process is dependent on the intensity of the exercise, as well as the type and frequency of the exercise. In a study conducted on mouse to ascertain the quantity of phosphocreatine reserved during exercises, it was proven that there was a significant difference between 24 hours and 72 hours recovery. The study analyzed that phosphocreatine reserves were higher during recovery with a duration of 72 hours [12]. This study analyzes the effect associated with recovery with regards to the protection of heart organs in overtraining conditions. The duration of recovery used in this study is same as those used in previous studies ( 72 hours or 3 days) and modification of extension of 7 (seven) days. The research examines $t$ the damages caused by myocardium in overtraining, as well as the proper effect of recovery in the exercise program, thereby, making this study to aim at analyzing the effect of recovery on myocardial damage as a result of overtraining during exercises. Obtained results from this study is likely to give birth to a step in the preparation of new protocols in the management and training of athletes against cardio vascular diseases.

\section{METHOD}

The methodology used in carrying out this research is the "randomized post-test only control group design". Data were collected at the end of the study by comparing the various outcomes in overtraining treated groups, overtraining with 3 (three) days' recovery and 7 (seven) days recovery with the untreated group (proportional exercise as control group). Four treatment groups were randomly created these are 1 (one) control group (proportional sport), 1 (one) group without recovery treatment overtraining, 1 (one) group overtraining treatment with recovery for 3 days and 1 (one) others group were given overtraining treatment a recovery period of 7 days. The study made use of male white rats (Wistar Ratus Norwegicus) between the ages of 3-4 months, and weighing between 180-220 grams. The research was approved by the Research Ethics Commission of Faculty of Medicine, of Sebelas Maret University No: 717 / VIII / HREC / 2016. The treatment given to mice was reinforced in a swimming pool of $50 \times 20 \times 60 \mathrm{~cm}$ (Giri, 2017). In the control group: the mice was allowed to swim in the afternoon for approximately 15 minutes after an hour of eating and drinking This was done throughout the days $(1,2,4,5,7$ every week). However, on the third and sixth day they were allowed to rest. The treatments were carried out for 8 weeks. At the end of the treatment, an autopsy was carried out on the mice to ascertain the condition of the blood and the heart organs. The overtraining treatment group was swum 2 (two) times daily (except on the 7 th day) until fatigue occurred and they could not swim again. The treatment was administered for 8 weeks. At the end of the treatment, an autopsy was immediately carried out on the mice to ascertain the blood and the heart organs The overtraining treatment group with 3 (three) recovery experienced the same treatment with the overtraining group and was given a rest period of 3 (three) days at the end of the treatment period. The overtraining treatment group with recovery 7 (seven) days, experienced the same treatment with the overtraining group and given a rest period of 7 (seven) days at the end of the treatment period an autopsy was carried out to ascertain the condition of the heart. Histologic preparations was done by making incisions in the heart muscle using microtome. The preparations were the stained with a Hematoxylin-Eosin (HE) staining technique to facilitate observation using a light microscope with 40 times magnification. This was used to assess hypertrophy, necrosis and increase the chromatin activities occurring in the myocardium. To detect the concentration of protein the mitochondria of MDA and SOD, the ELISA technique was used which precipitated antibodies as well. The data obtained were analyzed using the one-way ANOVA parametric test method followed by a Post Hoc Least Significant Difference (LSD) test.

\section{RESULTS AND DISCUSSION}

Based on the results of the study obtained, the mean MDA concentration of myocardium was found to be in the overtraining exercise group. It was found that the seven days recovery was lower than the control group (proportional sport) and those of the other two treatment groups with a control group mean MDA of $86.45 \mathrm{ng} / \mathrm{ml}$, with an overtraining group of $112.90 \mathrm{ng} / \mathrm{ml}$. The overtraining exercise group with a three-day recovery was found to be $109.53 \mathrm{ng} / \mathrm{ml}$ and the seven-day overtraining exercise group had a value of69.27 ng/ml.

The measurement result of SOD Miocardium concentration in this study showed that the mean SOD myocardium concentration in the control group (sport proportional) was higher than the other three treatment 
groups. The mean SOD myocardium control group was found to be $1516.05 \mathrm{ng} / \mathrm{ml}$, the overtraining exercise group was $829.94 \mathrm{ng} / \mathrm{ml}$, overtraining exercise with 3 (three) days recovery was $115,09 \mathrm{ng} / \mathrm{ml}$ and group of overtraining exercise with 7 (seven) days recovery was 939,44 ng/ml.

Description of observation and measurement result using a microscope showed that the overtraining exercise group with 3 (three) days recovery had the largest index of cardiomyocyte hypertrophy with a $77,625 \%$ compared to the mean result of the hypertrophy index control group which had a percentage of $26,5 \%$. The overtraining exercise group had a percentage of $75,5 \%$ and the overtraining group with 7 (seven) days recovery was $75.125 \%$.

Based on the observation of the anatomical pathology it can be seen that in the control group the index of myocardium necrosis was lower than the other three treatment groups. Signs of necrosis in the form of hypereosinophilic and curolisis, picnosis, curolisis, inflammatory cells and remnants cell was seen. The histopathological features of the necrosis are presented in following figure.

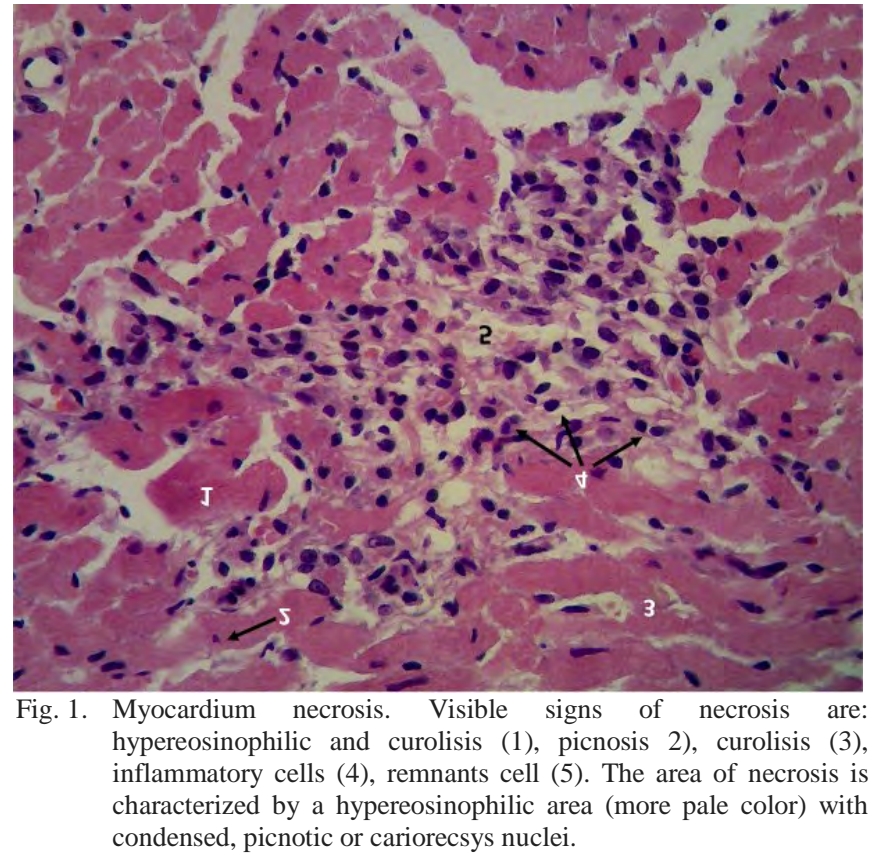

Observation results at the nuclei analyzed by an anatomical pathologist showed that the group of overtraining exercise with 7 (seven) days recovery timeframe, obtained the largest chromatin condensation index value of $83.8 \%$ compared to the chromatin condensation index rate of the control group which had a percentage value of $3.31 \%$, overtraining exercise group was $76.6 \%$ and the overtraining exercise group with 3 (three) days recovery was $78.7 \%$.

Comparative analysis was done on the posttest mean for each group. For the concentration of MDA myocardium, the results of Analysis Of Variance (ANOVA) showed $F=6,248$ with $\mathrm{p}=0,002$, resulting to the value of $\mathrm{p}$ being smaller than $\alpha=0,05$. Therefore, it can be concluded that there was an effect of overtraining exercise with three and seven days recovery on myocardium damage (MDA Myocardium levels). Result of Analysis of Variance (ANOVA) at mean of SOD Myocardium concentration with $\mathrm{F}=19,519$ and $\mathrm{p}=$ 0.0001 is concluded to have an impact on overtraining exercise with 7 (seven) day recovery to myocardium damage (SOD Myocardium levels). The result of Analysis of Variance (ANOVA) on the mean of Left Ventricular Hypertrophy Index was $F=140,732$ with $p=.0 .0001$. It can, therefore, be concluded that there was an effect of overtraining exercise with three and seven days recovery on Myocardium damage (Left Ventricular Hypertrophy Index). The result of Analysis of Variance (ANOVA) on the mean of Necrosis Index of Myocardium between groups, with $\mathrm{F}=$ 201,911 with $p=0.0001$ so it is concluded that there is an influence as a result of overtraining exercise on three and seven days recovery to the Myocardium Necrosis Index. Result of using the Analysis of Variance (ANOVA) on mean chromatin condensation index on cardiomyocyte between group shows $\mathrm{F}=524,466$ with $\mathrm{p}=0.0001$. It is, therefore, concluded that there is an influence on overtraining exercise with recovery of three and seven days to damage of myocardium (chromatin condensation index of cardiomyocyte)

Using One Way ANOVA (Analysis Of Variance) test, the differences between groups were found. This was then followed by LSD (Least Significant Difference) test which presented some of the following as a result:

1. The damage on myocardium is proportional to the amount of exercise, which is better than in the overtraining exercise. Although it has been given recovery because it resulted in lower MDA myocardium concentrations.

2. The condition of myocardium damage is proportional to the amount of exercise, and better than the overtraining exercise despite its given recovery state, since it shows the concentration of endogenous antioxidants (SOD) in the myocardium is higher.

3. The condition of myocardium damage in proportional exercise is better than the overtraining exercise load though it has been given recovery because it resulted in a lower left ventricular myocardium hypertrophy index.

4. The condition of myocardium damage is proportional to exercise and is better than the overtraining exercise despite its recovery state owing to its results in a lower Myocardium Necrosis Index.

5. Myocardium damage in proportional to exercise and it is better than the overtraining exercise despite its recovery timeframe. This is because it resulted in a lower cardiomyocyte chromatin condensation index.

\section{CONCLUSIONS}

The results of this study proves that there is no overtraining effect on myocardial damage in three and seven day recovery. Although recovery decreases such as MDA myocardium concentrations, increases SOD myocardium concentrations, it does not prevent left ventricular hypertrophy, necrosis and chromatin condensation 


\section{REFERENCE}

[1] H. Naci, J. P. A. Ioannidis. "Comparative effectiveness of exercise and drug interventions on mortality outcomes: meta epidemiological study," British Medical Journal. 2013;347.

[2] A. D'Silva, S. Sharma, "Exercise, the athlete's heart, and sudden cardiac death," The physician and sports medicine. 2014;42(2):100-13

[3] H-B. Kwak, "Aging, exercise, and extracellular matrix in the heart," Journal of exercise rehabilitation. 2013;9(3):338-347. doi:10.12965/jer.130049

[4] A. Pelliccia, R. Faqard, H. H. Bjornstad, A. Anastassakis, E. Arbustini, et al "Recommendations for competi- tive sports participation in athletes with cardiovascular disease: a consensus document from the Study Group of Sports Cardiology of the Working Group of Cardiac Rehabilitation and Exercise Physiology and the Working Group of Myocardial and Pericardial," Eur Heart J. 2005;26(14):1422-45.

[5] J. A. Hawley, M. Hargreaves, M. J. Joyner, J. R. Zierath. Integrative Biology of Exercise. Cell. 2014;159(4):738-49.

[6] A. C. Hackney, K. J. Koltun, "The Immune System And Overtraining In Athletes: Clinical Implications," Acta Clinica Croatia. 2014;51(4):633-41

[7] W. Stoiber, A. Obermayer, P Steinbacher, W. D. Krautgartner, The Role of Reactive Oxygen Species (ROS) in the Formation of Extracellular Traps (ETs) in Humans. Biomolecules. 2015;5(2):702-23.

[8] DP Marin, Ap Bolin, Tr Campoio, Ba Guerra, R Otton. "Oxidative Stress And Antioxidant Status Response Of Handball Athletes: Implications For Sport Training Monitoring," International Immunopharmacology. 2013;17(2):462-70

[9] R. Meeusen, M. Duclos, F. C. Foster, A. Fry, M. Gleeson, D Nieman, D Raglin, G Rietjens, J Steinacker, A Urhausen, "European College of Sport Science, American College of Sports Medicine. Prevention, Diagnosis, and Treatment of the Overtraining Syndrome: Joint Consensus Statement of the European College of Sport Science and the American College of Sports Medicine," Medicine \& Science in Sports \& Exercise. 2013;45(1):186-205.

[10] A. Pingitore, G. P. P. Lima, F. Mastorci, A. Quinones, G. Iervasi, C. Vassalle, "Exercise and oxidative stress: Potential effects of antioxidant dietary strategies in sports," Nutrition. 2015;31(7):91622

[11] K. G. Harmon, I. M. Asif, J. A. Drezner, "Incidence of Sudden Cardiac Death in National Collegiate Athletic Association Athlete," Circulation. 2011;123:1594-1600

[12] Harmon KG, Drezner JA, Wilson MG, Sharma S. Incidence of sudden cardiac death in athletes: a state- of-the-art review. British Journal of Sports Medicine. 2014;148:1185-92

[13] N. Chandra, R. Bastiaenen, M. Papadakis S. Sharma "Sudden Cardiac Death in Young Athletes: Practical Challenges and Diagnostic Dilemmas," Journal of the American College of Cardiology. 2013;61(10):1027-40.

[14] S. S. Chugh, J. B. Weiss, "Sudden Cardiac Death in the Older Athlete," Journal of the American College of Cardiology. 2015;65(5):493-502.

[15] SV de Noronha, S Sharma, M Papadakis, S Desai, G Whyte, Sheppard MN. Aetiology of sudden cardiac death in ath- letes in the United Kingdom: a pathological study. Heart. 2009;95(17):1409-14.

[16] Drezner JA, Harmon KG, Marek JC. Incidence of Sudden Cardiac Arrest in Minnesota High School Student Athletes: The Limitations of Catastrophic Insurance Claims. Journal of the American College of Cardiology. 2014;63(14):1455-6.

[17] OV Bogdanova, S Kanekar, KE D'Anci, PF Renshaw. Factors influencing behavior in the forced swim test. Physiology \& Behavior. 2013;118:227-39.

[18] A Can, DT Dao, M Arad, CE Terrillion, SC Piantadosi, TD Gould. The Mouse Forced Swim Test. Journal of Visualized Experiments. 2012(59):3638.

[19] A Oláh, BT Németh, C Mátyás, EM Horváth, L Hidi, E Birtalan, et al. Cardiac effects of acute exhaustive exercise in a rat model. International Journal of Cardiology.182:258-66.

[20] V Kumar, K Abbas, J Aster. Robbin and Cotran Basic Pathology. 9th ed. Canada: Elsevier Saunders; 2013. SLE 21 . Functional Capasity of The Cardiovascular System:
[21] Cardiac Output During Exercise. In: McArdle W, Katch F, Katch V, editors. Exercise Physiology. 7th ed. USA: Lippincott Williams and Wilkins; 2010. 